Article

\title{
A Three Function Variational Principle for Stationary Non-Barotropic Magnetohydrodynamics
}

\author{
Asher Yahalom 1,2,3 (D) \\ 1 Ariel University, Faculty of Engineering, Department of Electrical \& Electronic Engineering, Ariel 40700, \\ Israel; asya@ariel.ac.il \\ 2 Ariel University, Center for Astrophysics, Geophysics, and Space Sciences (AGASS), Ariel 40700, Israel; \\ 3 Princeton University, Princeton, New Jersey 08543, USA
}

\begin{abstract}
The current paper is devoted to the introduction of simpler Eulerian variational principles from which all the relevant equations of non-barotropic stationary magnetohydrodynamics can be derived for magnetic fields which lie on surfaces. A variational principle is given in terms of three independent variables for stationary non-barotropic magnetohydrodynamic flows. This is a smaller number of variables than the eight variables which appear in the standard equations of non-barotropic magnetohydrodynamics which are the magnetic field, the velocity field, the specific entropy and the density. We further investigate the case in which the flow along magnetic lines is not ideal.
\end{abstract}

Keywords: Magnetohydrodynamics, Variational Principles, Reduction of Variables

\section{Introduction}

Variational principles for magnetohydrodynamics (MHD) were introduced previously in both Lagrangian and Eulerian approaches. Sturrock [1] has studied in his book a Lagrangian variational formalism for MHD. Vladimirov and Moffatt [2] in a list of works have studied an Eulerian variational approach for incompressible MHD. However, their variational approach contained three more variables in addition to the seven functions which appear in the standard equations of incompressible MHD which are the magnetic field $\vec{B}$, velocity field $\vec{v}$ and pressure $P$. Kats [3] has extended Moffatt's work to compressible non barotropic flows but without minimizing the number of variables and thus the computational load. Moreover, Kats has demonstrated that the functions he suggested can be used to describe the motion of discontinuity surfaces [4,5]. Sakurai [6] has suggested a two function Eulerian variational principle for force-free magnetohydrodynamics and used it as a basis of a numerical scheme, his method is discussed [1]. A method of analyzing the equations for those two variables was suggested in [8]. Yahalom \& Lynden-Bell [9] combined the work of Sturrock [1] with the work of Sakurai [6] to obtain an Eulerian variational principle for barotropic MHD which depends on only six variables. The variational derivative of the suggested action produced all the equations needed to describe barotropic MHD without additional constraints. The equations obtained were similar to the ones of Frenkel, Levich \& Stilman [12] (and also to those of [13]). Yahalom [10] have shown that for barotropic MHD four functions suffice. Moreover, it was demonstrated that the discontinuities of some of those variables [11] are topological local conserved quantities.

Previous work was concerned with barotropic MHD in which the pressure and internal energy depend only on the density but not on the specific entropy and temperature. Variational principles of non barotropic MHD in which the internal energy and pressure are entropy dependent and thus temperature dependent effects can be described can be found in the work of Bekenstein \& Oron [14] in terms of 15 functions and V.A. Kats [3] in terms of 20 functions. We mention also the cases of 
incompressible flows in which density does not change in time or space and isochoric flows in which the volume of a system remains constant which are incompressible if the specific volume in the flow is also constant, those cases are beyond the scope of the current work. A. V. Kats in an outstanding paper [15] (section IV,E) has demonstrated that there is a large symmetry group (which may be considered a type of gauge freedom) associated with the choice of variables, it follows that the number of functions can be reduced. Yahalom [16,17] have shown that only five variables suffice to describe non barotropic MHD in the case that we require a Sakurai [6] form for the magnetic field. Morrison [7] has introduced a Hamiltonian formalism but this also depends on 8 canonical variables (see table 2 [7]). The paper of Yahalom [16] was concerned with general MHD non-stationary flows. In a subsequent paper [18] was concerned with stationary flows and introduced a 8 variable stationary variational principle, here we shall attempt to improve on this and obtain a 3 variable stationary variational principle for non-barotropic MHD. This will be done for a general case in which the magnetic field lines need not lie on entropy surfaces, for the restricted case in which the magnetic field lines lie on entropy surfaces see [19].

Applications of this paper may arise for both linear and non-linear stability analysis of stationary non barotropic MHD flows [26,28] and for designing numerical algorithms for integrating the equations of $\mathrm{MHD}[34,35,37]$. Another possible application is connected to obtaining new analytic solutions in terms of the variational functions [38] as will be described below.

The plan of this work is as follows: we introduce the standard notations and equations of non-barotropic magnetohydrodynamics for the stationary and non-stationary cases. Then we introduce the notions of load and metage. The variational principles follow.

\section{Standard formulation of non-barotropic magnetohydrodynamics}

The standard set of equations solved for non-barotropic magnetohydrodynamics are given below:

$$
\begin{gathered}
\frac{\partial \vec{B}}{\partial t}=\vec{\nabla} \times(\vec{v} \times \vec{B}), \\
\vec{\nabla} \cdot \vec{B}=0, \\
\frac{\partial \rho}{\partial t}+\vec{\nabla} \cdot(\rho \vec{v})=0, \\
\rho \frac{d \vec{v}}{d t}=\rho\left(\frac{\partial \vec{v}}{\partial t}+(\vec{v} \cdot \vec{\nabla}) \vec{v}\right)=-\vec{\nabla} p(\rho, s)+\frac{(\vec{\nabla} \times \vec{B}) \times \vec{B}}{4 \pi} . \\
\frac{d s}{d t}=0 .
\end{gathered}
$$

Here: $\frac{\partial}{\partial t}$ is the partial temporal derivative, $\frac{d}{d t}$ is the material derivative and $\vec{\nabla}$ is the standard nabla operator of vector calculus. $\vec{B}$ is the magnetic field, $\vec{v}$ is the velocity field, $\rho$ is the fluid mass density and $s$ is the specific entropy (entropy per unit mass). Finally $p(\rho, s)$ is the pressure which depends on both the density and entropy (and not just the density) through the thermodynamic equation of state (the non-barotropic case).

The justification for those equations can be found in standard books on MHD [1]. The above is valid for a collision-dominated plasma in local thermodynamic equilibrium. Such conditions are not always fulfilled by real physical plasmas, certainly not in astrophysics or in fusion-relevant magnetic confinement studies. Yet it is thought that the fastest macroscopic instabilities obey the above equations [11], while instabilities associated with viscous or finite conductivity terms take longer durations. According to a theorem by Bateman [39] every system can be described by a variational principle (including viscous plasma) the challenge is to discover a compact action functional which depends on a small amount of variational functions. This paper will discuss only ideal MHD while viscous MHD will be left for future endeavors. 
Equation (1) describes magnetic field lines that are moving with the fluid ("frozen" lines), equation (2) dictates a solenoidal field, equation (3) dictates mass conservation and equation (4) is the Euler equation for which pressure and Lorentz forces apply. The term:

$$
\vec{J}=\frac{\vec{\nabla} \times \vec{B}}{4 \pi},
$$

is the electric current density. Equation (5) is due to the lack of heat generation (null viscosity \& null resistivity) in ideal non-barotropic MHD and lack of heat conduction, thus the only remaining thermal process is heat convection. One needs to solve for eight variables $(\vec{v}, \vec{B}, \rho, s)$ eight equations $(1,3,4,5)$. Equation (2) is an initial condition on $\vec{B}$ field and is satisfied automatically later due to equation (1). For the stationary case we obtain:

$$
\begin{gathered}
\vec{\nabla} \times(\vec{v} \times \vec{B})=0, \\
\vec{\nabla} \cdot \vec{B}=0, \\
\vec{\nabla} \cdot(\rho \vec{v})=0, \\
\rho(\vec{v} \cdot \vec{\nabla}) \vec{v}=-\vec{\nabla} p(\rho, s)+\frac{(\vec{\nabla} \times \vec{B}) \times \vec{B}}{4 \pi} . \\
\vec{v} \cdot \vec{\nabla} s=0 .
\end{gathered}
$$

\section{Variational principle of non-barotropic MHD}

Here we generalize the analysis of [9] for the non-barotropic case. Let the action be:

$$
\begin{aligned}
A & \equiv \int \mathcal{L} d^{3} x d t \\
\mathcal{L} & \equiv \mathcal{L}_{1}+\mathcal{L}_{2} \\
\mathcal{L}_{1} & \equiv \rho\left(\frac{1}{2} \vec{v}^{2}-\varepsilon(\rho, s)\right)+\frac{\vec{B}^{2}}{8 \pi} \\
\mathcal{L}_{2} & \equiv v\left[\frac{\partial \rho}{\partial t}+\vec{\nabla} \cdot(\rho \vec{v})\right]-\rho \alpha \frac{d \chi}{d t}-\rho \beta \frac{d \eta}{d t}-\rho \sigma \frac{d s}{d t} \\
& -\frac{\vec{B}}{4 \pi} \cdot \vec{\nabla} \chi \times \vec{\nabla} \eta .
\end{aligned}
$$

$\varepsilon$ is the internal energy per mass. We recall the thermodynamic identities:

$$
\begin{aligned}
d \varepsilon= & T d s-P d \frac{1}{\rho}=T d s+\frac{P}{\rho^{2}} d \rho \\
& \frac{\partial \varepsilon}{\partial s}=T, \quad \frac{\partial \varepsilon}{\partial \rho}=\frac{P}{\rho^{2}} \\
w= & \varepsilon+\frac{P}{\rho}=\varepsilon+\frac{\partial \varepsilon}{\partial \rho} \rho=\frac{\partial(\rho \varepsilon)}{\partial \rho} \\
d w= & d \varepsilon+d\left(\frac{P}{\rho}\right)=T d s+\frac{1}{\rho} d P
\end{aligned}
$$

in the above $T$ is the temperature and $w$ is the specific enthalpy. in the above: $\varepsilon$ is the specific internal energy, $T$ is the temperature and $w$ is the specific enthalpy. A special case of equation of state is the polytropic equation of state [42]:

$$
p=K \rho^{\gamma}
$$

$K$ and $\gamma$ may depend on the specific entropy s. Hence:

$$
\frac{\partial \varepsilon}{\partial \rho}=K \rho^{\gamma-2} \Rightarrow \varepsilon=\frac{K}{\gamma-1} \rho^{\gamma-1}=\frac{p}{\rho(\gamma-1)} \Rightarrow \rho \varepsilon=\frac{p}{\gamma-1}
$$


the last identity is up to a function dependent on $s$. Obviously $v, \alpha, \beta, \sigma$ are Lagrange multipliers which were inserted in such a way that the variational principle will yield the following equations:

$$
\begin{aligned}
& \frac{\partial \rho}{\partial t}+\vec{\nabla} \cdot(\rho \vec{v})=0, \\
& \rho \frac{d \chi}{d t}=0, \\
& \rho \frac{d \eta}{d t}=0 . \\
& \rho \frac{d s}{d t}=0 .
\end{aligned}
$$

$v, \alpha, \beta, \sigma$ may be multiple valued. If $\rho$ does not vanish those are just the continuity equation (3), entropy conservation and a declaration that Sakurai's functions are moving with the flow. Varying with respect to $\vec{B}$ we obtain:

$$
\vec{B}=\hat{\vec{B}} \equiv \vec{\nabla} \chi \times \vec{\nabla} \eta
$$

Hence $\vec{B}$ is given in Sakurai's form and thus respects equation (2). It can be shown that if $\vec{B}$ is in the Sakurai's form, and equations (16) are fulfilled, then it follows that also equation (1) is respected.

We have shown that the entire set of equations of non-barotropic MHD is obtained from the above action except Euler's equations. We will now take care of that. Let us vary the above action with respect to $\vec{v}$ :

$$
\begin{aligned}
\delta_{\vec{v}} A & =\int d t\left\{\int d^{3} x d t \rho \delta \vec{v} \cdot[\vec{v}-\vec{\nabla} v-\alpha \vec{\nabla} \chi-\beta \vec{\nabla} \eta-\sigma \vec{\nabla} s]\right. \\
& \left.+\oint d \vec{S} \cdot \delta \vec{v} \rho v+\int d \vec{\Sigma} \cdot \delta \vec{v} \rho[v]\right\} .
\end{aligned}
$$

$\oint d \vec{S} \cdot \delta \vec{v} \rho v$ is zero in generic cases. For astrophysical scenarios $\rho=0$ on the boundary of the flow domain, in the case of a fluid contained in some pipe the conditions $\delta \vec{v} \cdot \hat{n}=0$ are imposed ( $\hat{n}$ denotes the unit vector normal to the boundary). $\int d \vec{\Sigma}$ on the cut of $v$ is zero if $v$ is single valued and $[v]=0$ which is the case for generic topologies. For $v$ which is multiple valued only a Kutta velocity variation [36] which is parallel to the cut will remove the cut integral. An arbitrary velocity variation on the cut dictates $\rho=0$ on the said surface which contradicts the notion that a cut is arbitrary as is the zero line of the azimuth. Later we demonstrate that the "cut" is co-moving thus it may become quite involved. This difficult reality may be more convenient to handle in symmetrical cases.

If the surface integrals vanish and also we have $\delta_{\vec{v}} A=0$ for an arbitrary velocity variation we find that:

$$
\vec{v}=\hat{\vec{v}} \equiv \vec{\nabla} v+\alpha \vec{\nabla} \chi+\beta \vec{\nabla} \eta+\sigma \vec{\nabla} s .
$$

Which resembles Clebsch form in non magnetic fluids [43,44]. Taking the variation with respect to $\rho$ we have:

$$
\begin{aligned}
\delta_{\rho} A & =\int d^{3} x d t \delta \rho\left[\frac{1}{2} \vec{v}^{2}-w-\frac{\partial v}{\partial t}-\vec{v} \cdot \vec{\nabla} v\right] \\
& +\int d t \oint d \vec{S} \cdot \vec{v} \delta \rho v+\int d t \int d \vec{\Sigma} \cdot \vec{v} \delta \rho[v] \\
& +\left.\int d^{3} x v \delta \rho\right|_{t_{0}} ^{t_{1}} .
\end{aligned}
$$


$w=\frac{\partial(\varepsilon \rho)}{\partial \rho}$ is the enthalpy per mass. Provided $\oint d \vec{S} \cdot \vec{v} \delta \rho v$ vanishes on the boundary and $\int d \vec{\Sigma} \cdot \vec{v} \delta \rho[v]$ vanishes on the cut of $v$ for $v$ which is multiple valued ${ }^{1}$ and in the beginning and the end the following equation results:

$$
\frac{d v}{d t}=\frac{1}{2} \vec{v}^{2}-w
$$

Since the right hand side of the equation is single valued since it is composed of physical variables which are not potentials, we obtain:

$$
\frac{d[v]}{d t}=0
$$

Thus the cut is co-moving with the flow and may become complicated. This situation may be less restrictive when the flow is symmetrical.

Finally we vary with respect to both $\chi$ and $\eta$ leading to the following expressions:

$$
\begin{aligned}
\delta_{\chi} A & =\int d^{3} x d t \delta \chi\left[\frac{\partial(\rho \alpha)}{\partial t}+\vec{\nabla} \cdot(\rho \alpha \vec{v})-\vec{\nabla} \eta \cdot \vec{J}\right] \\
& +\int d t \oint d \vec{S} \cdot\left[\frac{\vec{B}}{4 \pi} \times \vec{\nabla} \eta-\vec{v} \rho \alpha\right] \delta \chi \\
& +\int d t \int d \vec{\Sigma} \cdot\left[\frac{\vec{B}}{4 \pi} \times \vec{\nabla} \eta-\vec{v} \rho \alpha\right][\delta \chi] \\
& -\left.\int d^{3} x \rho \alpha \delta \chi\right|_{t_{0^{\prime}}} ^{t_{1}} \\
\delta_{\eta} A & =\int d^{3} x d t \delta \eta\left[\frac{\partial(\rho \beta)}{\partial t}+\vec{\nabla} \cdot(\rho \beta \vec{v})+\vec{\nabla} \chi \cdot \vec{J}\right] \\
& +\int d t \oint d \vec{S} \cdot\left[\vec{\nabla} \chi \times \frac{\vec{B}}{4 \pi}-\vec{v} \rho \beta\right] \delta \eta \\
& +\int d t \int d \vec{\Sigma} \cdot\left[\vec{\nabla} \chi \times \frac{\vec{B}}{4 \pi}-\vec{v} \rho \beta\right][\delta \eta] \\
& -\left.\int d^{3} x \rho \beta \delta \eta\right|_{t_{0}} ^{t_{1}} .
\end{aligned}
$$

If the temporal and boundary conditions are satisfied with respect to the variations $\delta \chi$ and $\delta \eta$ on the domain boundary and on the cuts for the case that some (or all) of the relevant variables are multiple valued, we obtain the following equations:

$$
\frac{d \alpha}{d t}=\frac{\vec{\nabla} \eta \cdot \vec{J}}{\rho}, \quad \frac{d \beta}{d t}=-\frac{\vec{\nabla} \chi \cdot \vec{J}}{\rho}
$$

in which equation (3) was used. By suitable temporal conditions we require that $\delta \eta$ and $\delta \chi$ vanish at initial and final times. Cases making the boundary term null include a boundary located at infinity in which both $\vec{B}$ and $\rho$ are null or a boundary which is impermeable and perfectly conducting. For the integral over the "cuts" to become null one can use $\delta \eta$ and $\delta \chi$ which are single valued. It is shown that $\chi$ can always be single valued, hence taking $\delta \chi$ to be single valued is not a restriction. In some topologies $\eta$ is multiple valued and for those cases a single valued $\delta \eta$ suffices to make the cut term vanish.

1 Thus either a Kutta type condition for the velocity in contradiction to the "cut" being an arbitrary surface, or a vanishing density variation on the same. 
Finally we take a variation of the action with respect to s:

$$
\begin{aligned}
\delta_{s} A & =\int d^{3} x d t \delta s\left[\frac{\partial(\rho \sigma)}{\partial t}+\vec{\nabla} \cdot(\rho \sigma \vec{v})-\rho T\right] \\
& +\int d t \oint d \vec{S} \cdot \rho \sigma \vec{v} \delta s-\left.\int d^{3} x \rho \sigma \delta s\right|_{t_{0}} ^{t_{1}}
\end{aligned}
$$

in which the temperature is $T=\frac{\partial \varepsilon}{\partial s}$. We notice that according to equation (19) $\sigma$ is single valued and hence no cuts are needed. Taking into account the continuity equation (3) we obtain for locations in which the density $\rho$ is not null the result:

$$
\frac{d \sigma}{d t}=T
$$

provided that $\delta_{s} A$ vanished for an arbitrary $\delta$ s.

\section{Euler's equations}

We shall now show that a velocity field given by equation (19), such that the equations for $\alpha, \beta, \chi, \eta, v, \sigma, s$ satisfy the corresponding equations $(16,21,25,27)$ must satisfy Euler's equations. Let us calculate the material derivative of $\vec{v}$ :

$$
\begin{aligned}
\frac{d \vec{v}}{d t} & =\frac{d \vec{\nabla} v}{d t}+\frac{d \alpha}{d t} \vec{\nabla} \chi+\alpha \frac{d \vec{\nabla} \chi}{d t}+\frac{d \beta}{d t} \vec{\nabla} \eta+\beta \frac{d \vec{\nabla} \eta}{d t} \\
& +\frac{d \sigma}{d t} \vec{\nabla} s+\sigma \frac{d \vec{\nabla} s}{d t} .
\end{aligned}
$$

It can be easily shown that:

$$
\begin{aligned}
\frac{d \vec{\nabla} v}{d t} & =\vec{\nabla} \frac{d v}{d t}-\vec{\nabla} v_{k} \frac{\partial v}{\partial x_{k}}=\vec{\nabla}\left(\frac{1}{2} \vec{v}^{2}-w\right)-\vec{\nabla} v_{k} \frac{\partial v}{\partial x_{k}} \\
\frac{d \vec{\nabla} \eta}{d t} & =\vec{\nabla} \frac{d \eta}{d t}-\vec{\nabla} v_{k} \frac{\partial \eta}{\partial x_{k}}=-\vec{\nabla} v_{k} \frac{\partial \eta}{\partial x_{k}} \\
\frac{d \vec{\nabla} \chi}{d t} & =\vec{\nabla} \frac{d \chi}{d t}-\vec{\nabla} v_{k} \frac{\partial \chi}{\partial x_{k}}=-\vec{\nabla} v_{k} \frac{\partial \chi}{\partial x_{k}} \\
\frac{d \vec{\nabla} s}{d t} & =\vec{\nabla} \frac{d s}{d t}-\vec{\nabla} v_{k} \frac{\partial s}{\partial x_{k}}=-\vec{\nabla} v_{k} \frac{\partial s}{\partial x_{k}}
\end{aligned}
$$

In which $x_{k}$ is a Cartesian coordinate and a summation convention is assumed. Inserting the result from equations $(29,16)$ into equation $(28)$ yields:

$$
\begin{aligned}
\frac{d \vec{v}}{d t} & =-\vec{\nabla} v_{k}\left(\frac{\partial v}{\partial x_{k}}+\alpha \frac{\partial \chi}{\partial x_{k}}+\beta \frac{\partial \eta}{\partial x_{k}}+\sigma \frac{\partial s}{\partial x_{k}}\right) \\
& +\vec{\nabla}\left(\frac{1}{2} \vec{v}^{2}-w\right)+T \vec{\nabla} s \\
& +\frac{1}{\rho}((\vec{\nabla} \eta \cdot \vec{J}) \vec{\nabla} \chi-(\vec{\nabla} \chi \cdot \vec{J}) \vec{\nabla} \eta) \\
& =-\vec{\nabla} v_{k} v_{k}+\vec{\nabla}\left(\frac{1}{2} \vec{v}^{2}-w\right)+T \vec{\nabla} s \\
& +\frac{1}{\rho} \vec{J} \times(\vec{\nabla} \chi \times \vec{\nabla} \eta) \\
& =-\frac{\vec{\nabla} p}{\rho}+\frac{1}{\rho} \vec{J} \times \vec{B} .
\end{aligned}
$$

Equation (19) and equation (17) are used. This shows that the non-barotropic MHD Euler equations can be obtained from the action of equation (12) and thus all the equations of non-barotropic MHD can 
be derived from the action for arbitrary volume variations restricted only on the relevant boundaries and cuts. Boundary variations serve to obtain boundary and initial conditions for the equations.

\section{Simplified action}

It might be argued that the above claims are misleading. It is declared that a simplified action for non-barotropic MHD is presented, instead six more functions $\alpha, \beta, \chi, \eta, v, \sigma$ are added to $\vec{B}, \vec{v}, \rho$, s. It will shown that the action described in equation (12) in a pedagogical form can be simplified. It is clear that the Lagrangian density of equation (12) can be written as:

$$
\begin{aligned}
\mathcal{L} & =-\rho\left[\frac{\partial v}{\partial t}+\alpha \frac{\partial \chi}{\partial t}+\beta \frac{\partial \eta}{\partial t}+\sigma \frac{\partial s}{\partial t}+\varepsilon(\rho, s)\right] \\
& +\frac{1}{2} \rho\left[(\vec{v}-\hat{\vec{v}})^{2}-(\hat{\vec{v}})^{2}\right] \\
& +\frac{1}{8 \pi}\left[(\vec{B}-\hat{\vec{B}})^{2}-(\hat{\vec{B}})^{2}\right]+\frac{\partial(v \rho)}{\partial t}+\vec{\nabla} \cdot(v \rho \vec{v}) .
\end{aligned}
$$

In which $\hat{\vec{v}}$ is a shorthand notation for $\vec{\nabla} v+\alpha \vec{\nabla} \chi+\beta \vec{\nabla} \eta+\sigma \vec{\nabla} s$ (see equation (19)) and $\hat{\vec{B}}$ is a shorthand notation for $\vec{\nabla} \chi \times \vec{\nabla} \eta$ (see equation (17)). Thus $\mathcal{L}$ has four contributions:

$$
\begin{aligned}
\mathcal{L} & =\hat{\mathcal{L}}+\mathcal{L}_{\vec{v}}+\mathcal{L}_{\vec{B}}+\mathcal{L}_{\text {boundary }} \\
\hat{\mathcal{L}} & \equiv-\rho\left[\frac{\partial v}{\partial t}+\alpha \frac{\partial \chi}{\partial t}+\beta \frac{\partial \eta}{\partial t}+\sigma \frac{\partial s}{\partial t}\right. \\
& \left.+\varepsilon(\rho, s)+\frac{1}{2}(\vec{\nabla} v+\alpha \vec{\nabla} \chi+\beta \vec{\nabla} \eta+\sigma \vec{\nabla} s)^{2}\right] \\
& -\frac{1}{8 \pi}(\vec{\nabla} \chi \times \vec{\nabla} \eta)^{2} \\
\mathcal{L}_{\vec{v}} & \equiv \frac{1}{2} \rho(\vec{v}-\hat{\vec{v}})^{2}, \\
\mathcal{L}_{\vec{B}} & \equiv \frac{1}{8 \pi}(\vec{B}-\hat{\vec{B}})^{2}, \\
\mathcal{L}_{\text {boundary }} & \equiv \frac{\partial(v \rho)}{\partial t}+\vec{\nabla} \cdot(v \rho \vec{v}) .
\end{aligned}
$$

The only term containing $\vec{v}$ is ${ }^{2} \mathcal{L}_{\vec{v}}$, after we equate to zero the variational derivative with respect to $\vec{v}$, we obtain equation (19) but will otherwise have no effect on the other variational derivatives. The only term depending on $\vec{B}$ is $\mathcal{L}_{\vec{B}}$ and this term will lead, after we set to zero the variational derivative, to equation (17) but will not change other variations. $\mathcal{L}_{\text {boundary }}$ contains only complete partial derivatives and thus does not contribute to the equations although it can influence the boundary conditions. Thus equations (16), equation (21), equations (25) and equation (27) are derived from:

$$
\begin{aligned}
& \hat{\mathcal{L}}[\alpha, \beta, \chi, \eta, v, \rho, \sigma, s]=-\rho\left[\frac{\partial v}{\partial t}+\alpha \frac{\partial \chi}{\partial t}+\beta \frac{\partial \eta}{\partial t}+\sigma \frac{\partial s}{\partial t}+\varepsilon(\rho, s)+\frac{1}{2}(\vec{\nabla} v+\alpha \vec{\nabla} \chi+\beta \vec{\nabla} \eta+\sigma \vec{\nabla} s)^{2}\right] \\
& \quad-\frac{1}{8 \pi}(\vec{\nabla} \chi \times \vec{\nabla} \eta)^{2}
\end{aligned}
$$

in which $\hat{\vec{v}}$ replaces $\vec{v}$ and $\hat{\vec{B}}$ replaces $\vec{B}$. After integrating the equations $(16,21,25,27)$ we are allowed to insert the potentials $\alpha, \beta, \chi, \eta, v, \sigma, s$ into equations (19) and (17) thus deriving the vector fields $\vec{v}$ and $\vec{B}$. To summarize, we have shown that the general ideal non-barotropic MHD problem is reduced from eight equations $(1,3,4,5)$ and the additional constraint $(2)$ to a problem of eight first order (in 
the temporal derivative) unconstrained equations. This set of equations can be derived from the Lagrangian density $\hat{\mathcal{L}}$.

\section{Stationary non-barotropic MHD}

Stationary configurations are unique to Eulerian fluid dynamics with no counter part in the Lagrangian description of fluid dynamics. Stationary configurations are defined by the fact that the fields $\vec{v}, \vec{B}, \rho, s$ are independent of time. This does not imply that the potentials $\alpha, \beta, \chi, \eta, v, \sigma$ are functions of only the spatial coordinates. Indedd choosing the potentials in such a way will lead to erroneous results such that the stationary equations of motion can not be derived from $\hat{\mathcal{L}}$ of equation (32). This problem can be solved as follows. Let us choose $\alpha, \beta, \chi, v, \sigma$ to depend on the spatial coordinates alone. $\eta$ is chosen such that:

$$
\eta=\bar{\eta}-t
$$

in which $\bar{\eta}$ depends on the spatial coordinates alone. $\hat{\mathcal{L}}$ of equation (32) will become:

$$
\hat{\mathcal{L}}=\rho(\beta-\varepsilon(\rho, s))-\frac{1}{2} \rho(\vec{\nabla} v+\alpha \vec{\nabla} \chi+\beta \vec{\nabla} \bar{\eta}+\sigma \vec{\nabla} s)^{2}-\frac{1}{8 \pi}(\vec{\nabla} \chi \times \vec{\nabla} \bar{\eta})^{2} .
$$

This can be compared with [2] equation 6.12 for incompressible flows in which their $I$ is analogue to our $\beta$. Notice that $\beta$ is not a conserved quantity but $I$ is conserved.

Varying the Lagrangian $\hat{L}=\int \hat{\mathcal{L}} d^{3} x$ with respect to $v, \alpha, \beta, \chi, \eta, \rho, \sigma, s$ leads to the following equations:

$$
\begin{aligned}
& \vec{\nabla} \cdot(\rho \hat{\vec{v}})=0, \\
& \rho \hat{\vec{v}} \cdot \vec{\nabla} \chi=0, \\
& \rho(\hat{\vec{v}} \cdot \vec{\nabla} \bar{\eta}-1)=0, \\
& \hat{\vec{v}} \cdot \vec{\nabla} \alpha=\frac{\vec{\nabla} \bar{\eta} \cdot \hat{\vec{J}}}{\rho}, \\
& \hat{\vec{v}} \cdot \vec{\nabla} \beta=-\frac{\vec{\nabla} \chi \cdot \overrightarrow{\vec{J}}}{\rho}, \\
& \beta=\frac{1}{2} \hat{\vec{v}}^{2}+w, \\
& \rho \hat{\vec{v}} \cdot \vec{\nabla} s=0, \\
& \rho \hat{\vec{v}} \cdot \vec{\nabla} \sigma=\rho T .
\end{aligned}
$$

Those lead to the stationary non-barotropic MHD equations:

$$
\begin{gathered}
\vec{\nabla} \times(\hat{\vec{v}} \times \hat{\vec{B}})=0, \\
\rho(\hat{\vec{v}} \cdot \vec{\nabla}) \hat{\vec{v}}=-\vec{\nabla} p(\rho, s)+\frac{(\vec{\nabla} \times \hat{\vec{B}}) \times \hat{\vec{B}}}{4 \pi} .
\end{gathered}
$$

In what follows we will attempt to reduce the number of variational variables from eight to four.

\section{Load and Metage}

The following section follows closely a similar section in [9]. Consider a thin tube surrounding a magnetic field line as described in figure 1 , the magnetic flux contained within the tube is:

$$
\Delta \Phi=\int \vec{B} \cdot d \vec{S}
$$




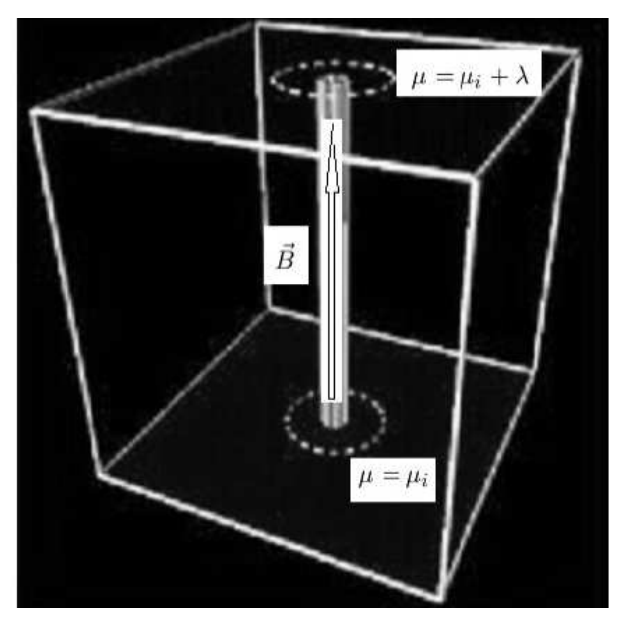

Figure 1. A thin tube surrounding a magnetic field line

and the mass contained with the tube is:

$$
\Delta M=\int \rho d \vec{l} \cdot d \vec{S}
$$

in which $d l$ is a short distance along the tube. Since the magnetic field lines are comoving as dictated by equation (1) and equation (3) both the quantities $\Delta \Phi$ and $\Delta M$ will not change during the motion of the tube. Since the tube is thin we may define the comoving magnetic load:

$$
\lambda=\frac{\Delta M}{\Delta \Phi}=\oint \frac{\rho}{B} d l
$$

calculated along the field line. The parts of the magnetic line which go out of the flow region to parts of space in which $\rho=0$ have zero contribution. $\lambda$ is single valued that can be experimentally measured in principle. Since $\lambda$ is comoving it satisfies:

$$
\frac{d \lambda}{d t}=0
$$

Also surfaces of constant magnetic load are comoving and are made from magnetic field lines. Hence the gradient to such surfaces must be normal to surface, and hence orthogonal to the field lines:

$$
\vec{\nabla} \lambda \cdot \vec{B}=0 .
$$

Consider an arbitrary comoving point on the field line denoted by $i$, consider another comoving point on the field line and mark it by $r$. The integral:

$$
\mu(r)=\int_{i}^{r} \frac{\rho}{B} d l+\mu(i)
$$

is also a comoving quantity which we denote after Lynden-Bell \& Katz [20] as the magnetic metage. $\mu(i)$ is a number which can be chosen differently for each line. Thus:

$$
\frac{d \mu}{d t}=0
$$

By differentiating along the field line we derive:

$$
\vec{\nabla} \mu \cdot \vec{B}=\rho .
$$

$\mu$ will be generally multiple valued. 


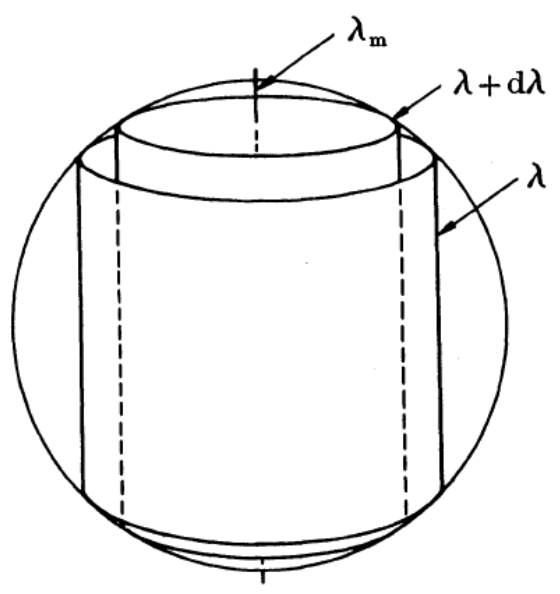

Figure 2. Constant load surfaces.

We now have two comoving coordinates of flow, $\lambda, \mu$ obviously in a three dimensional flow we should also have a third. However, before defining the third function we will first change $\lambda$ to a specific function of $\lambda$. Consider the magnetic flux within a load surface $\Phi(\lambda)$ described in figure 2 (the figure was also given in [20]). The magnetic flux is a comoving quantity and depends only on $\lambda$ of its boundary. Define:

$$
\chi=\frac{\Phi(\lambda)}{2 \pi}
$$

$\chi$ satisfies the equations:

$$
\frac{d \chi}{d t}=0, \quad \vec{B} \cdot \vec{\nabla} \chi=0
$$

Let us define $\eta^{*}$, since $\vec{\nabla} \mu$ is not orthogonal to $\vec{B}$ we can choose $\vec{\nabla} \eta^{*}$ to be orthogonal to $\vec{B}$ and not be in the direction of $\vec{\nabla} \chi$, we choose $\eta^{*}$ not to depend uniquely on $\chi$. Both $\vec{\nabla} \eta^{*}$ and $\vec{\nabla} \chi$ are orthogonal to $\vec{B}$, it must be that:

$$
\vec{B}=A \vec{\nabla} \chi \times \vec{\nabla} \eta^{*}
$$

However, using equation (2) we have:

$$
\vec{\nabla} \cdot \vec{B}=\vec{\nabla} A \cdot\left(\vec{\nabla} \chi \times \vec{\nabla} \eta^{*}\right)=0
$$

Which implies that $A$ is a function of $\chi, \eta^{*}$. Now we can define a new comoving function $\eta$ such that:

$$
\eta=\int_{0}^{\eta^{*}} A\left(\chi, \eta^{\prime *}\right) d \eta^{\prime *}, \quad \frac{d \eta}{d t}=0
$$

In terms of this function we obtain the Sakurai (Euler potentials) presentation:

$$
\vec{B}=\vec{\nabla} \chi \times \vec{\nabla} \eta
$$

We thus have shown how $\chi, \eta$ can be practically defined for a given $\vec{B}, \rho . \eta$ is defined in a non unique way since one can redefine $\eta$ by performing: $\eta \rightarrow \eta+f(\chi)$ in which $f(\chi)$ is any arbitrary function. $\chi, \eta$ serve as labels of the field lines. Moreover, we obtain the following expression for the magnetic flux:

$$
\Phi=\int \vec{B} \cdot d \vec{S}=\int d \chi d \eta
$$


In the case that the surface integral is performed inside a load contour we obtain:

$$
\Phi(\lambda)=\int_{\lambda} d \chi d \eta=\chi \int_{\lambda} d \eta=\left\{\begin{array}{c}
\chi[\eta] \\
\chi\left(\eta_{\max }-\eta_{\min }\right)
\end{array}\right.
$$

In one case the load surfaces are topological cylinders, $\eta$ is thus multiple valued and we obtain the upper value. In the second case the surfaces of constant load are topological spheres, $\eta$ is single valued and has both minimal $\eta_{\min }$ and maximal $\eta_{\max }$ values. Hence the lower value of is obtained. In some cases $\eta$ is identical to twice the latitude angle $\theta$. In those cases $\eta_{\min }=0$ (value at the "north pole") and $\eta_{\max }=2 \pi$ (value at the "south pole").

Comparing with equation (47) it follows that $\eta$ can be either single valued or multiple valued and that its discontinuity across its cut in the multiple valued scenario is $[\eta]=2 \pi$.

So far the discussion did not differentiate the cases of stationary and non-stationary flows. It should be noted that even for stationary flows one can have a non-stationary $\eta$ coordinates as the magnetic field depends only on the gradient of $\eta$ (see equation (52)), in particular if $\eta$ is stationary than $\eta+g(t)$ which is clearly not stationary will produce according to equation (52) a stationary magnetic field. In what follows we find it advantageous to use the form of $\eta$ given in equation (34) in which $\bar{\eta}$ is stationary.

$\chi, \eta, \mu$ are sufficient to label any fluid element in three dimensions. But for a non-barotropic ideal flow there is also another possible label $s$ which is comoving due to equation (5). The question then arises of the relation of $s$ to the other labels. Since we need to decide regarding the preferred set of labels it may seem that the physical labels are $\chi, \eta, s$ which are the surfaces on which the magnetic fields lie and the entropy. Thus $\mu$ is a function of $\chi, \eta, s$. If the magnetic field lines lie on entropy surface, $\mu$ is an independent label. The density is:

$$
\rho=\frac{\partial \mu}{\partial s} \frac{\partial(\chi, \eta, s)}{\partial(x, y, z)} .
$$

$\mu$ can be defined for each line separately according to equation (44), it is thus obvious that such a choice exist in which $\mu$ is uniquely a function of $s$. One may also think of the entropy $s$ as a functions $\chi, \eta, \mu$. In what follows we shall ignore the status of $s$ as a label and consider it as a variational function which only attains a status of a label at the variational extremum.

\section{A Simpler variational principle of stationary non-barotropic magnetohydrodynamics}

In a previous paper [18] we have shown that stationary non-barotropic magnetohydrodynamics can be described in terms of eight first order differential equations and by an action principle from which those equations can be derived. Below we will show that one can do better for the case in which the magnetic field lines lie on an entropy surface, in this case three functions will suffice to describe stationary non-barotropic magnetohydrodynamics.

Consider equation (48), for a stationary flow it takes the form:

$$
\vec{v} \cdot \vec{\nabla} \chi=0
$$

Hence $\vec{v}$ can take the form:

$$
\vec{v}=\frac{\vec{\nabla} \chi \times \vec{K}}{\rho} .
$$

However, the velocity field must satisfy the stationary mass conservation equation (3):

$$
\vec{\nabla} \cdot(\rho \vec{v})=0
$$


A sufficient condition (although not necessary) for $\vec{v}$ to be a solution of equation (58) is that $\vec{K}$ is of the form $\vec{K}=\vec{\nabla} N$, here $N$ is arbitrary. Thus, $\vec{v}$ can be written as:

$$
\vec{v}=\frac{\vec{\nabla} \chi \times \vec{\nabla} N}{\rho}
$$

Let us now calculate $\vec{v} \times \vec{B}$ in which $\vec{B}$ is given by Sakurai's presentation equation (52):

$$
\begin{aligned}
\vec{v} \times \vec{B} & =\left(\frac{\vec{\nabla} \chi \times \vec{\nabla} N}{\rho}\right) \times(\vec{\nabla} \chi \times \vec{\nabla} \eta) \\
& =\frac{1}{\rho} \vec{\nabla} \chi(\vec{\nabla} \chi \times \vec{\nabla} N) \cdot \vec{\nabla} \eta .
\end{aligned}
$$

$N$ can be at most a function of the three coordinates $\chi, \mu, \bar{\eta}$, hence:

$$
\vec{\nabla} N=\frac{\partial N}{\partial \chi} \vec{\nabla} \chi+\frac{\partial N}{\partial \mu} \vec{\nabla} \mu+\frac{\partial N}{\partial \bar{\eta}} \vec{\nabla} \bar{\eta}
$$

Inserting equation (61) into equation (60) will yield:

$$
\vec{v} \times \vec{B}=\frac{1}{\rho} \vec{\nabla} \chi \frac{\partial N}{\partial \mu}(\vec{\nabla} \chi \times \vec{\nabla} \mu) \cdot \vec{\nabla} \bar{\eta} .
$$

Rearranging terms and using equation (52) we can write:

$$
\vec{v} \times \vec{B}=-\frac{1}{\rho} \vec{\nabla} \chi \frac{\partial N}{\partial \mu}(\vec{\nabla} \mu \cdot \vec{B}) .
$$

However, using equation (46) this will simplify to the form:

$$
\vec{v} \times \vec{B}=-\vec{\nabla} \chi \frac{\partial N}{\partial \mu}
$$

Inserting equation (64) into equation (7) will lead to the equation:

$$
\vec{\nabla}\left(\frac{\partial N}{\partial \mu}\right) \times \vec{\nabla} \chi=0
$$

However, since $N$ is at most a function of $\chi, \mu, \bar{\eta}$ it follows that $\frac{\partial N}{\partial \mu}$ is some function of $\chi$ :

$$
\frac{\partial N}{\partial \mu}=-F(\chi)
$$

This can be easily integrated to yield:

$$
N=-\mu F(\chi)+G(\chi, \bar{\eta})
$$

Inserting $N$ into equation (59) will result in:

$$
\vec{v}=\frac{\vec{\nabla} \chi \times\left(-F(\chi) \vec{\nabla} \mu+\frac{\partial G}{\partial \bar{\eta}} \vec{\nabla} \bar{\eta}\right)}{\rho} .
$$

We shall replace $\chi, \bar{\eta}$ with $\chi^{\prime}, \bar{\eta}^{\prime}$ defined as:

$$
\chi^{\prime}=\int F(\chi) d \chi, \quad \bar{\eta}^{\prime}=\frac{\bar{\eta}}{F(\chi)} .
$$


This will not affect equation (52) as:

$$
\vec{B}=\vec{\nabla} \chi \times \vec{\nabla} \eta=\vec{\nabla} \chi \times \vec{\nabla} \bar{\eta}=\vec{\nabla} \chi^{\prime} \times \vec{\nabla} \bar{\eta}^{\prime} .
$$

However, the velocity will take the simpler form:

$$
\vec{v}=\frac{\vec{\nabla} \chi^{\prime} \times \vec{\nabla}\left(-\mu+G^{\prime}\left(\chi^{\prime}, \bar{\eta}^{\prime}\right)\right)}{\rho}
$$

$G^{\prime}=\frac{G}{F}$. We recall that $\mu$ is defined in equation (44) up to constant which may vary between field lines. As the lines are labelled by their $\chi^{\prime}, \bar{\eta}^{\prime}$ values we can add a function of $\chi^{\prime}, \bar{\eta}^{\prime}$ to $\mu$ without effecting its desired properties. We thus define $\mu^{\prime}$ of the form:

$$
\mu^{\prime}=\mu-G^{\prime}\left(\chi^{\prime}, \bar{\eta}^{\prime}\right)
$$

$\mu^{\prime}$ can be non single-valued. Inserting equation (72) into equation (71) will lead to:

$$
\vec{v}=\frac{\vec{\nabla} \mu^{\prime} \times \vec{\nabla} \chi^{\prime}}{\rho}
$$

The primes on $\chi, \mu, \bar{\eta}$ will be ignored from now on. This is analogues to [2] equation 7.11 for incompressible flows, our $\mu$ and $\chi$ resemble their $A$ and $\Psi$. $\vec{v}$ satisfies the following:

$$
\vec{v} \cdot \vec{\nabla} \mu=0, \quad \vec{v} \cdot \vec{\nabla} \chi=0, \quad \vec{v} \cdot \vec{\nabla} \bar{\eta}=1,
$$

where we have used equation (45) and equation (52). $\mu, \chi$ are both comoving and stationary, $\bar{\eta}$ satisfies equation (34). If:

$$
\text { basis }=(\vec{\nabla} \chi, \vec{\nabla} \bar{\eta}, \vec{\nabla} \mu),
$$

is a local vector basis at any point in space than their exists a dual basis:

$$
\text { dual basis }=\frac{1}{\rho}(\vec{\nabla} \bar{\eta} \times \vec{\nabla} \mu, \vec{\nabla} \mu \times \vec{\nabla} \chi, \vec{\nabla} \chi \times \vec{\nabla} \bar{\eta})=\left(\frac{\vec{\nabla} \bar{\eta} \times \vec{\nabla} \mu}{\rho}, \vec{v}, \frac{\vec{B}}{\rho}\right) .
$$

Such that:

$$
\text { basis }_{i} \cdot \text { dual basis }_{j}=\delta_{i j}, \quad i, j \in[1,2,3],
$$

$\delta_{i j}$ is Kronecker's delta. The surfaces $\chi, \mu, \bar{\eta}$ generate a local vector basis for space, while the physical fields of interest $\vec{v}, \vec{B}$ are contained in the dual basis. We can now construct a vector product of $\vec{v}$ and $\vec{B}$ and taking into account equations $(73,52)$ we arrive at the equation:

$$
\vec{v} \times \vec{B}=\vec{\nabla} \chi
$$

thus $\vec{v}$ and $\vec{B}$ lie on $\chi$ surfaces and are a vector basis for this two dimensional surface. This can be compared with [2] equation 5.6 for incompressible flows, the $J$ appearing in that paper is analogue to $\chi$. 


\section{A three function variational principle for stationary MHD}

Previously we demonstrated that provided that the $\vec{v}$ is given by equation (73) and $\vec{B}$ is given by equation (52) than equations $(7,8,9)$ are satisfied. To complete the set of equations we will show how the Euler equations (4) can be derived from the action:

$$
\begin{aligned}
A & \equiv \int \mathcal{L} d^{3} x d t \\
\mathcal{L} & \equiv \rho\left(\frac{1}{2} \vec{v}^{2}-\varepsilon(\rho, s)\right)-\frac{\vec{B}^{2}}{8 \pi}
\end{aligned}
$$

in which both $\vec{v}$ and $\vec{B}$ are given by equation (73) and equation (52) respectively and the density $\rho$ is given by equation (45):

$$
\rho=\vec{\nabla} \mu \cdot \vec{B}=\vec{\nabla} \mu \cdot(\vec{\nabla} \chi \times \vec{\nabla} \eta)=\frac{\partial(\chi, \eta, \mu)}{\partial(x, y, z)} .
$$

The Lagrangian density of equation (79) takes the more explicit form:

$$
\mathcal{L}[\chi, \eta, \mu]=\rho\left(\frac{1}{2}\left(\frac{\vec{\nabla} \mu \times \vec{\nabla} \chi}{\rho}\right)^{2}-\varepsilon(\rho, s(\chi, \eta, \mu))\right)-\frac{(\vec{\nabla} \chi \times \vec{\nabla} \eta)^{2}}{8 \pi}
$$

and can be seen explicitly to depend on only three functions. We underline that if the magnetic field lines lie on entropy surfaces. $s$ must be a function of $\chi, \eta$ only and does not depend on $\mu$. Let us make arbitrary small variations $\delta \alpha_{i}=(\delta \chi, \delta \eta, \delta \mu)$ of the functions $\alpha_{i}=(\chi, \eta, \mu)$. Let us define a $\Delta$ variation that does not modify the $\alpha_{i}$ 's, such that:

$$
\Delta \alpha_{i}=\delta \alpha_{i}+(\vec{\xi} \cdot \vec{\nabla}) \alpha_{i}=0,
$$

in which $\vec{\xi}$ is the Lagrangian displacement, thus:

$$
\delta \alpha_{i}=-\vec{\nabla} \alpha_{i} \cdot \vec{\xi}
$$

Which will lead to the equation:

$$
\vec{\xi} \equiv-\frac{\partial \vec{r}}{\partial \alpha_{i}} \delta \alpha_{i}
$$

Making a variation of $\rho$ given in equation (80) with respect to $\alpha_{i}$ will yield:

$$
\delta \rho=-\vec{\nabla} \cdot(\rho \vec{\xi})
$$

Making a variation of $s$ will result in:

$$
\delta s=\frac{\partial s}{\partial \alpha_{i}} \delta \alpha_{i}=-\frac{\partial s}{\partial \alpha_{i}} \vec{\nabla} \alpha_{i} \cdot \vec{\xi}=-\vec{\nabla} s \cdot \vec{\xi}
$$

Furthermore, taking the variation of $\vec{B}$ given by Sakurai's representation (52) with respect to $\alpha_{i}$ will yield:

$$
\delta \vec{B}=\vec{\nabla} \times(\vec{\xi} \times \vec{B}) .
$$

It remains to calculate $\delta \vec{v}$ by varying equation (73) this will yield:

$$
\delta \vec{v}=-\frac{\delta \rho}{\rho} \vec{v}+\frac{1}{\rho} \vec{\nabla} \times(\rho \vec{\xi} \times \vec{v}) .
$$


Varying the action will result in:

$$
\begin{aligned}
\delta A & =\int \delta \mathcal{L} d^{3} x d t \\
\delta \mathcal{L} & =\delta \rho\left(\frac{1}{2} \vec{v}^{2}-w(\rho, s)\right)-\rho T \delta s+\rho \vec{v} \cdot \delta \vec{v}-\frac{\vec{B} \cdot \delta \vec{B}}{4 \pi}
\end{aligned}
$$

Inserting equations $(85,87,88)$ into equation $(89)$ will yield:

$$
\begin{aligned}
\delta \mathcal{L} & =\vec{v} \cdot \vec{\nabla} \times(\rho \vec{\xi} \times \vec{v})-\frac{\vec{B} \cdot \vec{\nabla} \times(\vec{\xi} \times \vec{B})}{4 \pi}-\delta \rho\left(\frac{1}{2} \vec{v}^{2}+w\right)+\rho T \vec{\nabla} s \cdot \vec{\xi} \\
& =\vec{v} \cdot \vec{\nabla} \times(\rho \vec{\xi} \times \vec{v})-\frac{\vec{B} \cdot \vec{\nabla} \times(\vec{\xi} \times \vec{B})}{4 \pi}+\vec{\nabla} \cdot(\rho \vec{\xi})\left(\frac{1}{2} \vec{v}^{2}+w\right) \\
& +\rho T \vec{\nabla} s \cdot \vec{\xi} .
\end{aligned}
$$

Using the well known vector identity:

$$
\vec{A} \cdot \vec{\nabla} \times(\vec{C} \times \vec{A})=\vec{\nabla} \cdot((\vec{C} \times \vec{A}) \times \vec{A})+(\vec{C} \times \vec{A}) \cdot \vec{\nabla} \times \vec{A}
$$

and the theorem of Gauss we can write now equation (89) in the form:

$$
\begin{aligned}
\delta A & =\int d t\left\{\oint d \vec{S} \cdot\left[\rho(\vec{\xi} \times \vec{v}) \times \vec{v}-\frac{(\vec{\xi} \times \vec{B}) \times \vec{B}}{4 \pi}+\left(\frac{1}{2} \vec{v}^{2}+w\right) \rho \vec{\xi}\right]\right. \\
& \left.+\int d^{3} x \vec{\xi} \cdot\left[\rho \vec{v} \times \vec{\omega}+\vec{J} \times \vec{B}-\rho \vec{\nabla}\left(\frac{1}{2} \vec{v}^{2}+w\right)+\rho T \vec{\nabla} s\right]\right\} .
\end{aligned}
$$

Time integration is of no consequence in the above. Notice that we used equation (6) and the vorticity $\vec{\omega}=\vec{\nabla} \times \vec{v}$. If $\delta A=0$ for $\vec{\xi}$ nullifying the boundary term but otherwise arbitrary, then:

$$
\rho \vec{v} \times \vec{\omega}+\vec{J} \times \vec{B}-\rho \vec{\nabla}\left(\frac{1}{2} \vec{v}^{2}+w\right)+\rho T \vec{\nabla} s=0 .
$$

Using the well known vector identity:

$$
\frac{1}{2} \vec{\nabla}\left(\vec{v}^{2}\right)=(\vec{v} \cdot \vec{\nabla}) \vec{v}+\vec{v} \times(\vec{\nabla} \times \vec{v})
$$

and rearranging terms we recover the stationary Euler equation:

$$
\rho(\vec{v} \cdot \vec{\nabla}) \vec{v}=-\vec{\nabla} p+\vec{J} \times \vec{B}
$$

\section{An Application: Helical Stratified Magnetic Field}

\subsection{The Magnetic Field and Related Labels}

Consider [48] a magnetohydrodynamic flow of uniform density $\rho$. Furthermore assume that the flow contains a helical stratified magnetic field:

$$
\vec{B}= \begin{cases}2 B_{\perp}\left(1-\frac{R}{a}\right) \hat{\phi}+B_{z 0} \hat{z} & R<a \\ 0 & R>a\end{cases}
$$


$R, \phi, z$ are cylindrical coordinates, $\hat{R}, \hat{\phi}, \hat{z}$ are unit vectors and $B_{z 0}, B_{\perp}$ are constants. The field is confined to a cylinder of Radius $a$ and does not depend on $z$. We assume that $z=0$ and $z=L$ are identified such that a topological torus is realized. The only field lines that will be closed are such that:

$$
\frac{n}{m}=\frac{B_{\perp}}{\pi R B_{z 0}}\left(1-\frac{R}{a}\right) L, \quad n, m \text { integers }
$$

lines not satisfying this condition are surface filling. The field lines lie on cylindrical surfaces, so one can calculate the flux through a circular surface lying on the plane $z$ and bounded by $R$. Thu flux is:

$$
\Phi=\int \vec{B} \cdot d \vec{S}=\pi R^{2} B_{z 0}
$$

The $\chi$ function can now be calculated according to equation (47) to yield the value:

$$
\chi=\frac{1}{2} B_{z 0} R^{2}
$$

Solving equation (17) for $\eta$ we obtain the following non unique solution:

$$
\eta=\phi-\frac{2 B_{\perp}}{B_{z 0}}\left(1-\frac{R}{a}\right) \frac{z}{R} .
$$

Finally we solve equation (46) for $\mu$, here we suggest the following simple and non unique solution:

$$
\mu=\frac{\rho}{B_{z 0}} z
$$

Thus, $\mu$ surfaces are just $z$ planes. Notice that since we have identified the planes $z=0$ and $z=L, \mu$ is non single valued. The same can be said on $\eta$ which is doubly non-single valued in both the $z$ and $\phi$ directions.

\subsection{The Velocity Field}

A stationary velocity field $\vec{v}$ must satisfy equation (7) and equation (9). Such a velocity field can be constructed using the labels $\mu$ and $\chi$ (see equation (73)):

$$
\vec{v}=k \frac{\vec{\nabla} \mu \times \vec{\nabla} \chi}{\rho} .
$$

$k$ is a dimensional constant that we will choose such that $k=\frac{v_{0}}{a}$. Plugging in $\mu$ from equation (101) and $\chi$ from equation (99) we arrive at the simple expression:

$$
\vec{v}=v_{0} \frac{R}{a} \hat{\phi}
$$

This expression can be shown to solve equation (1) and equation (3) by direct substitution. Equation (10) can be solved by the pressure function:

$$
p(R)=\rho\left[\frac{B_{\perp}^{2}}{\pi}\left(3 \frac{R}{a}-\frac{R^{2}}{a^{2}}-\ln \left(\frac{R}{a}\right)-2\right)+\frac{1}{2} v_{0}^{2}\left(\frac{R^{2}}{a^{2}}-1\right)\right], \quad p(a)=0 .
$$




\section{The three function action principle for a static configuration}

The static configuration is a stationary flow such that $\vec{v}=0$. In this case the mass conservation equation (7) and magnetic field equation (9) are satisfied trivially. To complete the set of equations we will show how the static Euler equations (4) can be derived from the action:

$$
\begin{aligned}
& A \equiv-\int \mathcal{L} d^{3} x d t \\
& \mathcal{L} \equiv \rho \varepsilon(\rho, s)+\frac{\vec{B}^{2}}{8 \pi}
\end{aligned}
$$

in which $\vec{B}$ is given by equation (52) and the density $\rho$ is given by equation (80). The Lagrangian density of equation (105) can be put in the more explicit form:

$$
\mathcal{L}[\chi, \eta, \mu]=\rho \varepsilon(\rho, s(\chi, \eta, \mu))+\frac{(\vec{\nabla} \chi \times \vec{\nabla} \eta)^{2}}{8 \pi}
$$

Varying the action will result in:

$$
\begin{aligned}
\delta A & =-\int \delta \mathcal{L} d^{3} x d t \\
\delta \mathcal{L} & =\delta \rho(w(\rho, s))+\rho T \delta s+\frac{\vec{B} \cdot \delta \vec{B}}{4 \pi}
\end{aligned}
$$

Inserting equations $(85,87)$ into equation $(107)$ will yield:

$$
\begin{aligned}
\delta \mathcal{L} & =\frac{\vec{B} \cdot \vec{\nabla} \times(\vec{\xi} \times \vec{B})}{4 \pi}+\delta \rho w+\rho T \vec{\nabla} s \cdot \vec{\xi} \\
& =\frac{\vec{B} \cdot \vec{\nabla} \times(\vec{\xi} \times \vec{B})}{4 \pi}-\vec{\nabla} \cdot(\rho \vec{\xi}) w+\rho T \vec{\nabla} s \cdot \vec{\xi} .
\end{aligned}
$$

Using the well known vector identity (91), and the theorem of Gauss we can write now equation (107) in the form:

$$
\begin{aligned}
\delta A & =\int d t\left\{\oint d \vec{S} \cdot\left[-\frac{(\vec{\xi} \times \vec{B}) \times \vec{B}}{4 \pi}+w \rho \vec{\xi}\right]\right. \\
& \left.+\int d^{3} x \vec{\xi} \cdot[\vec{J} \times \vec{B}-\rho \vec{\nabla} w+\rho T \vec{\nabla} s]\right\} .
\end{aligned}
$$

The time integration is of no consequence in the above expression. We used equation (6). If $\delta A=0$ for a arbitrary $\vec{\xi}$ but such that the boundary term is null, then we obtain:

$$
\vec{J} \times \vec{B}-\rho \vec{\nabla} w+\rho T \vec{\nabla} s=0 .
$$

and rearranging terms we recover the stationary Euler equation:

$$
\vec{J} \times \vec{B}-\vec{\nabla} p=0
$$

\section{Transport phenomena}

In many plasmas including static configurations heat is transferred preferably along magnetic field lines:

$$
\vec{J}_{H}=-\hat{k} \vec{\nabla} T
$$

in which $\hat{k}$ is a tensor of heat conductivity. This tensor is usually larger in the magnetic field direction and thus can be written as:

$$
\hat{k}=k_{\perp}(I-\hat{b} \otimes \hat{b})+k_{\|} \hat{b} \otimes \hat{b}
$$


in the above $\hat{b} \equiv \frac{\vec{B}}{B}$ is a unit vector in the magnetic field direction, $\otimes$ is the tensor product, $I$ is the unit matrix, $k_{\perp}$ is the heat conductivity in directions perpendicular to magnetic field lines and $k_{\|}$is the larger heat conductivity in the direction parallel to magnetic field lines. The equation for a stationary heat flux configuration is:

$$
\vec{\nabla} \cdot \vec{J}_{H}=0 \Rightarrow \vec{\nabla} \cdot(\hat{k} \vec{\nabla} T)=0 .
$$

This equation can be derived from the heat Lagrangian and Lagrangian density:

$$
L_{H} \equiv \int \mathcal{L}_{H} d^{3} x, \quad \mathcal{L}_{H} \equiv \frac{1}{2}(\vec{\nabla} T)^{t} \hat{k} \vec{\nabla} T=\frac{1}{2} \partial_{i} T \hat{k}_{i j} \partial_{j} T,
$$

in the above $(\vec{\nabla} T)^{t}$ is the transpose of the $\vec{\nabla} T, \partial_{i} \equiv \frac{\partial}{\partial x_{i}}$ and Einstein summation convention is assumed. Taking the variation with respect to the temperature $T$ yields:

$$
\delta \mathcal{L}_{H}=(\vec{\nabla} T)^{t} \hat{k} \vec{\nabla} \delta T,
$$

hence:

$$
\delta L_{H}=\int d^{3} x[\vec{\nabla} \cdot(\hat{k} \vec{\nabla} T \delta T)-\delta T \vec{\nabla} \cdot(\hat{k} \vec{\nabla} T)],
$$

and using the theorem of Gauss:

$$
\delta L_{H}=\int d \vec{S} \cdot(\hat{k} \vec{\nabla} T) \delta T-\int d^{3} x \delta T \vec{\nabla} \cdot(\hat{k} \vec{\nabla} T),
$$

thus for appropriate boundary conditions we derive equation (114). We notice that heat conduction is not taken into account in ideal MHD which only assumes convection of heat. However, provided that conduction is seen as a secondary process with respect to convection we may obtain using the ideal variational principle a stationary or static magnetic field configuration using the appropriate variational expression given in previous sections. And then using the known magnetic field configuration we derive the appropriate heat flux transport using $L_{H}$.

\section{Conclusion}

It is shown that stationary non-barotropic magnetohydrodynamics can be derived from a variational principle of three functions. We have shown this for both the stationary and static cases.

Possible applications include stability studies of stationary MHD and development of numerical methods for integrating MHD equations. It may be possible to incorporate the current formalism in existing codes instead of developing a new code from scratch. Possible codes are described in [21-25]. We anticipate applications of this work to linear and non-linear stability studies of known MHD configurations [26-28]. For achieving this we may need to add additional constants of motion constraints to the action $[29,30]$ see also [31-33]. As for designing numerical methods for integrating the equations of MHD one may follow the approach described in [34-37].

Another application of the variational variables is obtaining analytic solutions for the MHD equations. Although the equations are very difficult to solve being both partial differential equations and nonlinear, possible solutions can be found using variational functions. An example for this approach is the self gravitating torus described in [38] and also in section 10.

One can use continuous symmetries which appear in the action to derive through Noether theorem new conservation laws. An example for such derivation can be found in [40] and [48].

Topological invariants have always been useful, and there are such invariants in MHD flows. For example the two helicities (magnetic helicity and cross helicity) have long been useful in research into the problem of hydrogen fusion, and in various astrophysical scenarios. In previous studied $[9,11,45]$ relations between helicities and symmetries of MHD were made. Also the functions of the current action are helpful for identifying and characterizing new topological invariants in MHD [46-48]. 
Although ideal MHD does not describe fully real plasmas, we show here how processes such as heat conduction can be also described using variational analysis provided that the magnetic field configuration is given approximately by ideal variational analysis.

To conclude we underline the limitations of the current work. First MHD is a fluid theory and as such misses some of the processes that can only be addressed by a detailed kinetic model. Second ideal MHD considered here neglects important non ideal processes such as resistive heating, heat conduction and viscous effects. Moreover, we assume magnetic field that lie on surfaces (that is equation (17)) while for some MHD configurations magnetic field lines are volume filling, for such cases the current approach is not applicable.

Acknowledgments: The author wishes to thank Professor Allan Reiman of Princeton University for discussions and helpful comments and for suggesting some of the sections in the current paper.

1. P. A. Sturrock, Plasma Physics (Cambridge University Press, Cambridge, 1994)

2. V. A. Vladimirov and H. K. Moffatt, J. Fluid. Mech. 283 125-139 (1995)

3. A. V. Kats, Los Alamos Archives physics-0212023 (2002), JETP Lett. 77, 657 (2003)

4. A. V. Kats and V. M. Kontorovich, Low Temp. Phys. 23, 89 (1997)

5. A. V. Kats, Physica D 152-153, 459 (2001)

6. T. Sakurai, Pub. Ast. Soc. Japan 31209 (1979)

7. P.J. Morrison, Poisson Brackets for Fluids and Plasmas, AIP Conference proceedings, Vol. 88, Table 2.

8. W. H. Yang, P. A. Sturrock and S. Antiochos, Ap. J., 309383 (1986)

9. A. Yahalom and D. Lynden-Bell, "Simplified Variational Principles for Barotropic Magnetohydrodynamics," (Los-Alamos Archives physics/0603128) Journal of Fluid Mechanics, Vol. 607, 235-265, 2008.

10. Yahalom A., "A Four Function Variational Principle for Barotropic Magnetohydrodynamics" EPL 89 (2010) 34005, doi: 10.1209/0295-5075/89/34005 [Los - Alamos Archives - arXiv: 0811.2309]

11. Asher Yahalom "Aharonov - Bohm Effects in Magnetohydrodynamics" Physics Letters A. Volume 377, Issues 31-33, 30 October 2013, Pages 1898-1904.

12. A. Frenkel, E. Levich and L. Stilman Phys. Lett. A 88, p. 461 (1982)

13. V. E. Zakharov and E. A. Kuznetsov, Usp. Fiz. Nauk 40, 1087 (1997)

14. J. D. Bekenstein and A. Oron, Physical Review E Volume 62, Number 4, 5594-5602 (2000)

15. A. V. Kats, Phys. Rev E 69, 046303 (2004)

16. A. Yahalom "Simplified Variational Principles for non-Barotropic Magnetohydrodynamics". (arXiv: 1510.00637 [Plasma Physics]) J. Plasma Phys. (2016), vol. 82, 905820204. doi:10.1017/S0022377816000222.

17. Asher Yahalom "Non-Barotropic Magnetohydrodynamics as a Five Function Field Theory". International Journal of Geometric Methods in Modern Physics, No. 10 (November 2016). Vol. 131650130 (13 pages) @World Scientific Publishing Company.

18. A. Yahalom "Simplified Variational Principles for Stationary non-Barotropic Magnetohydrodynamics" International Journal of Mechanics, Volume 10, 2016, p. 336-341. ISSN: 1998-4448.

19. Asher Yahalom "A Simpler Variational Principle for Stationary non-Barotropic Ideal Magnetohydrodynamics". Chaotic Modeling and Simulation (CMSIM), 1: 19-33, 2018. Received: 15 October 2017 / Accepted: 28 December 2017.

20. D. Lynden-Bell and J. Katz "Isocirculational Flows and their Lagrangian and Energy principles", Proceedings of the Royal Society of London. Series A, Mathematical and Physical Sciences, Vol. 378, No. 1773, 179-205 (Oct. 8, 1981).

21. Mignone, A., Rossi, P., Bodo, G., Ferrari, A., \& Massaglia, S. (2010). High-resolution 3D relativistic MHD simulations of jets. Monthly Notices of the Royal Astronomical Society, 402(1), 7-12.

22. Miyoshi, T., \& Kusano, K. (2005). A multi-state HLL approximate Riemann solver for ideal magnetohydrodynamics. Journal of Computational Physics, 208(1), 315-344.

23. Igumenshchev, I. V., Narayan, R., \& Abramowicz, M. A. (2003). Three-dimensional magnetohydrodynamic simulations of radiatively inefficient accretion flows. The Astrophysical Journal, 592(2), 1042.

24. Faber, J. A., Baumgarte, T. W., Shapiro, S. L., \& Taniguchi, K. (2006). General relativistic binary merger simulations and short gamma-ray bursts. The Astrophysical Journal Letters, 641(2), L93. 
25. Hoyos, J., Reisenegger, A., \& Valdivia, J. A. (2007). Simulation of the Magnetic Field Evolution in Neutron Stars. In VI Reunion Anual Sociedad Chilena de Astronomia (SOCHIAS) (Vol. 1, p. 20).

26. V. A. Vladimirov, H. K. Moffatt and K. I. Ilin, J. Fluid Mech. 329, 187 (1996); J. Plasma Phys. 57, 89 (1997); J. Fluid Mech. 390, 127 (1999)

27. Bernstein, I. B., Frieman, E. A., Kruskal, M. D., \& Kulsrud, R. M. (1958). An energy principle for hydromagnetic stability problems. Proceedings of the Royal Society of London. Series A. Mathematical and Physical Sciences, 244(1236), 17-40.

28. J. A. Almaguer, E. Hameiri, J. Herrera, D. D. Holm, Phys. Fluids, 31, 1930 (1988)

29. V. I. Arnold, Appl. Math. Mech. 29, 5, 154-163.

30. V. I. Arnold, Dokl. Acad. Nauk SSSR 162 no. 5.

31. J. Katz, S. Inagaki, and A. Yahalom, "Energy Principles for Self-Gravitating Barotropic Flows: I. General Theory", Pub. Astro. Soc. Japan 45, 421-430 (1993).

32. Yahalom A., Katz J. \& Inagaki K. 1994, Mon. Not. R. Astron. Soc. 268 506-516.

33. A. Yahalom, "Stability in the Weak Variational Principle of Barotropic Flows and Implications for Self-Gravitating Discs". Monthly Notices of the Royal Astronomical Society 418, 401-426 (2011).

34. A. Yahalom, "Method and System for Numerical Simulation of Fluid Flow", US patent 6,516,292 (2003).

35. A. Yahalom, \& G. A. Pinhasi, "Simulating Fluid Dynamics using a Variational Principle", proceedings of the AIAA Conference, Reno, USA (2003).

36. A. Yahalom, G. A. Pinhasi and M. Kopylenko, "A Numerical Model Based on Variational Principle for Airfoil and Wing Aerodynamics", proceedings of the AIAA Conference, Reno, USA (2005).

37. D. Ophir, A. Yahalom, G.A. Pinhasi and M. Kopylenko "A Combined Variational and Multi-Grid Approach for Fluid Dynamics Simulation" Proceedings of the ICE - Engineering and Computational Mechanics, Volume 165, Issue 1, 01 March 2012, pages 3 -14 , ISSN: 1755-0777, E-ISSN: 1755-0785.

38. Asher Yahalom "Using fluid variational variables to obtain new analytic solutions of self-gravitating flows with nonzero helicity" Procedia IUTAM 7 (2013) 223 - 232.

39. H. Bateman "On Dissipative Systems and Related Variational Principles" Phys. Rev. 38, 815 Published 15 August 1931.

40. Asher Yahalom, "A New Diffeomorphism Symmetry Group of Magnetohydrodynamics" V. Dobrev (ed.), Lie Theory and Its Applications in Physics: IX International Workshop, Springer Proceedings in Mathematics \& Statistics 36, p. 461-468, 2013.

41. Katz, J. \& Lynden-Bell, D. Geophysical \& Astrophysical Fluid Dynamics 33,1 (1985).

42. Binney J. \& Tremaine S., 1987, Galactic Dynamics, Princeton University Press

43. Clebsch, A., Uber eine allgemeine Transformation der hydrodynamischen Gleichungen.

J. reine angew. Math. 1857, 54, 293-312.

44. Clebsch, A., Uber die Integration der hydrodynamischen Gleichungen. J. reine angew. Math. 1859, 56, 1-10.

45. A. Yahalom, "Helicity Conservation via the Noether Theorem" J. Math. Phys. 36, 1324-1327 (1995). [Los-Alamos Archives solv-int/9407001]

46. Asher Yahalom "A Conserved Local Cross Helicity for Non-Barotropic MHD" (ArXiv 1605.02537). Pages 1-7, Journal of Geophysical \& Astrophysical Fluid Dynamics. Published online: 25 Jan 2017. Vol. 111, No. 2, 131-137.

47. Asher Yahalom "Non-Barotropic Cross-helicity Conservation Applications in Magnetohydrodynamics and the Aharanov - Bohm effect" (arXiv:1703.08072 [physics.plasm-ph]). Fluid Dynamics Research, Volume 50, Number 1, 011406. https://doi.org/10.1088/1873-7005/aa6fc7 . Received 11 December 2016, Accepted Manuscript online 27 April 2017, Published 30 November 2017.

48. Asher Yahalom \& Hong Qin "Noether Currents for Eulerian Variational Principles in Non-Barotropic Magnetohydrodynamics and Topological Conservations Laws" Journal of Fluid Mechanics, 908, A4. doi:10.1017/jfm.2020.856, 2021. 